(liquide complexe) diminue très vite quand la température s'élève ; mais cette diminution s'amortit elle-même assez vite, ce que l'on

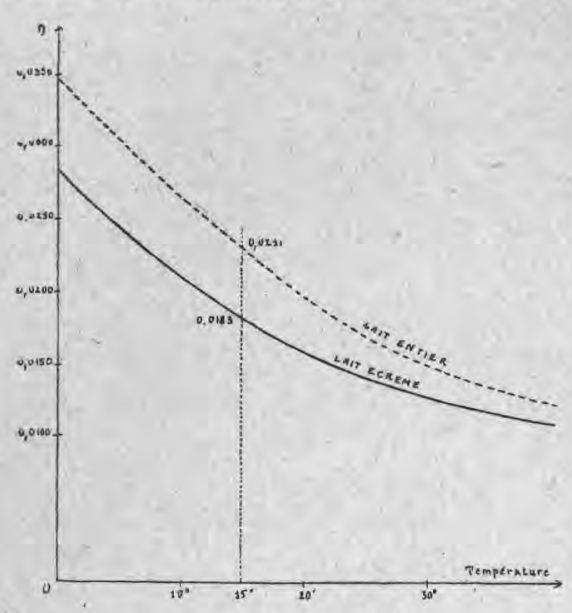

Fig. 4

Variations de la viscosité du lait avec la température. peut représenter par une courbe en forme d'hyperbole (voir figure 4).

Il est donc indispensable d'opérer exactement à la même température, si l'on veut comparer la viscosité de deux laits. Notons d'autre part l'importance d'une élévation de température comme cause favorisante dans l'écrémage du lait.

Ces quelques observations sur la viscosité du lait doivent être complétées pour nous permettre d'apprécier les limites de variation du coefficient de viscosité, qui sont en rapport avec un certain nombre de facteurs. D'après les résultats déjà obtenus, il est facile de prévoir l'influence possible de la constitution physico-chimique, mais surtout de la composition chimique, et par conséquent de toutes les causes de variation de cette composition (mélange des laits, période de lactation, affections mammaires, rétention lactée, etc.). C'est dans cette voie que nous désirons poursuivre l'étude ainsi commencée.

\title{
CARACTĖRES BIOLOGIQUES ET CULTURAUX DE L'AGENT ÉTIOLOGIQUE ORDINAIRE DE LA MAMIMITE STREPTOCOCCIQUE DE LA VACHE
}

\author{
par J. M. ROSELL, M. D.
}

Chef du Département de Bactériologie à l'Institut agricole d'Oka et à l'Ecols de Médecine vétérinaire de la Province de Québec.

La mammite streptococcique est une maladie des vaches laitières, qui a tout d'abord attiré l'attention des vétérinaires et des bureaux d'hygiène des pays du nord et du centre de l'Europe, et qui maintenant intéresse tous les pays.

C'est une maladie qui, actuellement, oceasionne aux troupeaux laitiers des pertes économiques plus grandes peut-être que la tuberculose et l'avortement, qui sont deux des maladies les plus importantes affectant les berceaux de l'Industrie laitière. 
Différents pays ont calculé que la perte en lait occasionnée par la mammite représente à peu près le tiers de la production totale, et les chiffres donnés par différents pays comme argent perdu par la mammite se traduisent en monnaie par centaines de millions par année pour beaucoup de pays.

Des études étendues concernant cette affection ont été faites en différents laboratoires d'Europe, spécialement dans les dernières huit ou dix années, et aussi aux Etats-Unis, depuis les trois dernières années.

$$
*^{*} *
$$

Grẩce à l'aide du Ministère d'Agriculture de la Provinee de Québec et du National Research Council du Canada, le Département de Bactériologie de l'Institut agricole d'Oka et del'Ecole de Médecine vétérinaire de la Province de Québec, a pu, depuis cinq ans, entreprendre une étude systématique, que nous continuons encore, sur cette maladie. Cette étude a porté, jusqu'à maintenant, sur de nombreuses fermes de différents comtés de la Province de Québec, et, aux Etats-Unis, dans quelques fermes de l'Etat de New-York et de Virginia, en ce dernier endroit avec la coopération du Bureau de l'Industrie animale de Washington ( $\mathrm{D}^{\mathrm{r}}$ W. T. MTLLER).

L'objectif principal de nos études, dont les résultats ont été donnés dans les publications annotées dans la bibliographie, comprenait les points suivants :

a) Etendue de la maladie, qui, dans la majorité des cas, surtout au début, ne sé dépiste que par les procédés de laboratoire ;

b) Altérations chimiques et biologiques, macro et microscopiques, que la maladie produit dans le lait et qui peuvent permettre un diagnostic dès le commencement de la maladie;

c) Agent étiologique de la maladie ;

d) Sa prévention et son traitement.

Dans cette exposition, nous nous sommes limités exclusivement à décrire les caractéristiques biologiques et culturaux du germe que nous considérons être en relation étiologique principale avec cette maladie.

Les chercheurs qui ont travaillé sur la mammite, reconnaissent, à la presque unanimité, le streptocoque désigné sous les noms de streptococcus mastitidis et de streptococcus agalactice, comme l'agent étiologique de cette maladie. Cela n'empêche pas, qu'en certains cas, d'autres types de streptocoques aient été trouvés, exclusivement, ou associés au streptococcus mastitidis, dans différents quartiers malades.

Mais, selon notre expérience résultant de l'étude individuelle de plusieurs milliers d'échantillons de lait, prélevés aseptiquement des 
quartiers séparés du pis de la vache, nous pouvons affirmer que, dans au moins $98 \%$ des cas, nous avons toujours isolé le même type de streptocoque. Ce streptocoque présente des caractères assez constants et distincts de ceux des autres streptocoques considérés actuellement comme des espèces ou types fixes de streptocoques, et il peut, par conséquent, être considéré comme une individualité ou espèce définie, différenciable des plus proches formes streptococciques apparentées, desquelles il se différencie toujours par plusieurs caractères biologiques et culturaux.

De même que dans toutes les espèces microbiennes, on trouve pour ce streptocoque, des formes atypiques ou intermédiaires, rarement cependant.

Les caractères distinctifs culturaux et biologiques de ce streptocoque qui, dans les travaux taxonomiques, est désigné sous le nom de streptococcus mastitidis (NocARD MoLlEREAU), ou Strep, agalactiae contagiosa (KITT), sont ceux que nous résumons dans ce travail, quant aux points essentiels. Nous avons établi ces caractéristiques au cours d'une étude faite sur plus de 400 souches isolées par nous et nos assistants F. TroudEL et Ed. BROCHU, ainsi que dans des travaux faits en collaboration avec le $D^{r}$ W. T. MrLLER, du Département d'Agriculture de Washington, et, à l'Université de McGill, en colla-

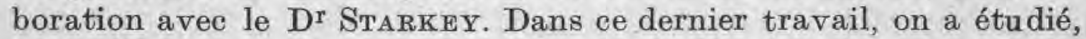
en même temps qu'une trentaine de souches de streptocoques de la mammite provenant de différents instituts européens et américains, une série de nouvelles souches récemment isolées à côté de 78 souches de streptocoques humains et d'animaux de différentes origines. Les résultats de cette étude, non encore publiés et présentés en première communication au Congrès de la Section de laboratoire de l'Association Canadienne d'Hygiène publique tenu à Toronto en décembre 1933 et au Congrès de la Société des Bactériologistes américains de décembre 1933 à Philadelphie, sont presque entièrement conformes aux résultats publiés dans des travaux antérieurs et que nous résumons ici.

Nous devons ajouter que ces caractères concordent presque en entier avec ceux trouvés par d'autres chercheurs qui se sont occupés de cette maladie en quelques laboratoires européens et récemment aussi en d'autres de ce continent.

\section{ASPECT MICROSCOPIQUE DU LAIT}

Les streptocoques de la mammite peuvent se présenter, dans le sédiment du lait ou dans le lait, tantôt sous forme de longues chaînes, quelquefois composées de plusieurs centaines de germes, dans un lait enrichi, tantôt sous forme de courtes chaînes, tantôt aussi, et nous voulons faire remarquer ce point, sous forme de diplocoques 
isolés et même d'amas très difficiles à distinguer des microcoques saprophytes ordinaires de la mamelle, presque toujours présents, même dans le lait de mammite. Souvent les streptocoques sont trouvés à l'intérieur des leucocytes qui les ont phagocytés. Bien souvent aussi, l'examen d'un seul étalement de sédiment ne permet pas de découvrir un seul streptocoque, bien que, par ensemencement à partir du même sédiment, on ait pu les découvrir. Notre expérience nous montre qu'au moyen de la calture, nous sommes capables de découvrir les streptocoques 15 à 25 fois (pour cent) plus souvent que par le seul examen microscopique.

\section{ÉTUDE PAR LA CULTURE}

L'étude par les cultures est Je procédé vraiment bactériologique. Nous donnons ci-après les procédés que notre expérience nous a montrés comme les plus pratiques pour arriver à l'isolement et à la caractérisation des streptocoques de la mammite. Nous conseillons la marche suivante :

Préparer des plaques de Pírin avec l'un ou l'autre des quatre types de gélose suivants :

a) Agar ou gélose ordinaire de bouillon de viande glucosé ;

b) Agar-sang ou agar-sérum ;

c) Agar à base d'albumine-alcaline-sérum-saccharose-bromocrésol-pourpre, appelé aussi «Klimmer-agar ».

Les streptocoques se développent bien sur chacun de ces types d'agar, mais spécialement sur les deux derniers.

L'agar-sang a encore l'avantage de fournir, tout en faisant les eultures, les renseignements voulus sur l'action hémolysante ou non des streptocoques isolés. Au point de vue médical et sanitaire, l'agar-sang aurait l'avantage de pouvoir éventuellement faire connaître l'existence des streptocoques pyogenes hémolytiques, qui eausent parfois la mammite, seuls ou associés aux streptocoques de la mammite ordinaire, et aussi l'existence des streptocoques appelés épidémiques, décrits 15 fois en Amérique comme produisant des épidémies d'angines.

L'agar "Klimmer " a le grand avantage d'être un très bon milieu pour le streptocoque et de permettre en même temps de différencier, par le changement de couleur qu'il produit, les streptocoques de la mammite d'autres streptocoques, qui, éventuellement, pourraient se trouver dans le lait, surtout s'il n'a pas été prélevé aseptiquement.

On peut déjà différencier les colonies de streptocoques parfois après 12 heures de séjour à l'étuve et toujours après 24 heures. On peut, avec un peu de pratique, distinguer facilement les colonies de streptocoques de celles des autres bactéries quiéventuellement peuvent 
être présentes dans le lait. Dans un lait pris aseptiquement et directement dans des tubes stériles, en évitant toute contamination extérieure, les seules autres bactéries qui peuvent cultiver généralement à côté des streptocoques sont les microcoques de la mamelle et diphtéroïdes.

Les microcoques saprophytes de la mamelle, qui malheureusement contaminent trop souvent les cultures, sont un peu difficiles à distinguer, dans les premières 24-48 heures, des streptocoques. A partir du troisième jour, ces colonies, ordinairement plus blanchâtres et jaunâtres, plus proéminentes et plus grandes, plus opaques et plus sèches que celles des streptocoques, sont facilement reconnaissables eomme colonies de microcoques. Dans les plaques de gélose de KurmMER, on peut différencier les streptocoques des microcoques, parce que, chez les premiers, les colonies et la zone voisine du milieu prennent une couleur jaune doré pendant que les colonies de microcoques et celles des autres bactéries, autant qu'elles n'acidifient pas le sucrose, ne changent pas la couleur violacée du milieu. Malheureusement; assez souvent, dans une même colonie, les streptocoques et les microcoques se développent à la fois.

Dans des cultures faites avec du lait qui n'a pas été prélevé avec assez de précautions, il peut se développer généralement ou des bacilles provenant des spores portées par la poussière des étables, ou des bactéries lactiques, ou des colibacilles ou d'autres microbes. Les colonies plus étendues ou ordinairement plus sèches des bacilles, celles du $B$. coli, plus irrégulières en leur contour, plus élevées, plus opaques et plus grandes, et celles des bactéries lactiques semblables à des pointes d'aiguilles, peuvent en général être facilement distinguées des colonies de streptocoques.

Nous décrirons maintenant les aspects morphologiques les plus caractéristiques que le streptocoque de la mammite présente dans les différents milieux de culture, ainsi que les changements qu'il produit dans les milieux dont on se sert pour faire sa caractérisation biologique, et partant, sa classification ou détermination comme variété particulière de streptocoque.

\section{ASPECT DES COLONIES SUR AGAR NUTRITIF ORDINAIRE}

La colonie est ronde, à bords légèrement granulés, transparents, avec le centre légèrement plus foncé, et ordinairement pas plus grande qu'un millimètre après 48 heures de culture. Les colonies sont parfois plus petites ( $0 \mathrm{~mm} .5)$, et rarement plus grandes que $2 \mathrm{~mm}$. Ordinairement, dans un agar humide, le bord qu'on observe au microscope est légèrement granuleux et quelque peu irrégulier. Au toucher avec l'anse de platine, la colonie paraît avoir une consistance - mucoïde. Toutes les colonies ne présentent pas toujours le même 
aspect. Quelques colonies, surtout celles qui sont produites avec un sédiment provenant de lait mammiteux dont les streptocoques sont difficiles à cultiver, s'étendent d'une façon irrégulière dans leur conformation comme dans leurs dimensions. Il est possible que cette irrégularité de la forme des colonies et aussi la difficulté de cultiver, en certains cas, des streptocoques du lait mammiteux, soient dues à une action bactériophagique ou autre.

\section{ASPECTS MORPHOLOGIQUES DES STREPTOCOQUES DE LA MAMMITE SUR DIFFERENTS MILIEUX}

La forme de chaque individu des streptocoques de la mammite, dans son groupement en chaînes longues ou courtes, ou en amas, comme cela arrive le plus souvent en milieu solide, est presque sans exception la forme de cocci aplatis, deux à deux, l'un contre l'autre, étant plus allongés dans l'un de leurs diamètres que dans l'autre, donnant l'aspect d'un double disque ou d'un grain de café. Les formes sphériques trouvées chez quelques individus, faisant partie d'une chaîne de formes typiques aplaties ou d'un amas de streptocoques, se sont montrées comme des formes hypertrophiées et ne sont en somme qu' une phase de la prédivision ou des dissociations morphologiques individuelles.

Ordinairement les cellules se divisent en plans parallèles à angle droit de la direction de croissance, de sorte que, les cellules demeurant attachées l'une à l'autre, il se forme des chaînes plus ou moins longues, quelques-unes pouvant être formées de plusieurs milliers d'individus, quand le milieu de eulture leur est favorable. Parfois la division a lieu dans deux directions différentes, comme chez les microcoques.

L'aspect morphologique des streptocoques provenant de colonies d'agar correspond fréquemment à celui des microcoques ou staphylocoques. Le streptocoque des colonies ordinaires apparaît rarement sous forme de longueś chaînes. Les cultures en bouillon présentent ordinairement l'arrangement en chaînes de dimensions variables. La morphologie individuelle des streptocoques peut varier en oultures successives. On doit toujours compter sur la possibilité, - cas d'ailleurs assez rare, croyons-nous, - de voir des cultures pures de streptocoques contenir différentes variétés susceptibles de prendre différents aspects culturaux dans les sous-cultures. Rappelons-nous aussi $q$ u'une souche de streptocoques peut se dissocier dans les sous-cultures en souches d'aspects différents. D'autre part les microcoques du pis contaminent très souvent les cultures apparemment pures du streptocoque de la mammite, même après plusieurs sous-cultures.

CARACTERISTIQUES BIOLOGIQUES DU STREPTOCOQUE DE LA MAMIMITE ISOLE PENDANT NOS ÉTUDES

Le streptocoque de la mammite, perdant facilement sa vitalité, 
ne se conserve pas longtemps en culture pure. Il se conserve cependant des mois dans un agar-sérum recouvert d'huile paraffinée et sous forme de piqûre en gélatine.

Culture en bouillon. - Dans un bouillon glucosé ou lactosé, d'un $p \mathrm{H} \quad 7,2-7,4$, après 16-24 heures, le streptocoque produit un sédiment floconneux, laissant au-dessus un liquide clair et transparent. Mais il y a quelques cultures du streptocoque de la mammite qui, en certaines circonstances, troublent le bouillon. Ils prennent dans ces cas la forme des types diplocoques ou chaînes courtes. Un trouble, après une incubation de 24 heures, dénote toujours la formation de chaînes courtes, ou la contamination par le microcoque, tandis qu'un sédiment floconneux, laissant un bouillon clair, indique la présence de longues chaînes. La nature et le $p \mathrm{H}$ du bouillon ont une influence sur l'aspect de la culture.

Lait tournesolé. (7-10\% de teinture de tournesol.) - Le lait tournesolé est un des meilleurs milieux de culture pour différencier le streptocoque de la mammite des autres sortes comme les S. Tactique et pyogène. Le streptocoque de la mammite, en culture jeune, dans un lait tournesolé à $37^{\circ} \mathrm{C}$., produit les changements typiques suivants : $1^{\circ} \mathrm{il} \mathrm{acidifie}$ et rougit ce lait en 10 à 24 heures ; $2^{\circ}$ le lait donne en 20 à 24 heures un coagulum consistant et homogène, avec ou sans la présence d'un peu de sérum, et souvent avec la formation de stries. $A u$ fond du tube de culture, apparaît souvent une légère réduction du tournésol, surtout si la colonne de lait est élevée (10 à $15 \mathrm{~cm}$.). Le streptocoque lactique coagule d'abord le lait, puis le décolore avec une légère rougeur en haut du tube. Il est assez rare que d'autres sortes de streptocoques produisent des changements dans le lait tournesolé exactement comme ceux qu'on trouve avec le streptocoque de la mammite et le streptocoque lactique. Si cela arrive, parfois les autres caractères culturaux permettront le diagnostic différentiel. Mais quelques races du streptocoque de la mammite peuvent produire, après deux ou trois jours d'incubation, une réduction plus ou moins prononcée du lait tournesolé, d'autres n'en produisent que très rarement ou ne coagulent pas le lait. Nous avons eu des souches dissociées qui, en cultures successives, ont coagulé de différentes façons et avee réduction.

Les streptocoques cultivés souvent sur des milieux artificiels ne conservent pas toujours leurs caractères en ce qui concerne, entre autres, l'acidification et la coagulation du lait tournesolé. De vieilles cultures des germes de la mammite streptococcique, qui ont perdu plus ou moins leur pouvoir de coaguler le lait, ont plus ou moins recouvré leur activité après plusieurs passages en milieu lait. Le lait tournesolé desséché ne semble pas donner des réactions aussi typiques que le lait tournesolé ordinaire. 
Lait avec bleu de méthylène. (1: 200.000.) - Le streptocoque de la mammite ne produit aucun changement dans ce lait, lequel est réduit et coagulé par le streptocoque lactique. D'autres sortes de streptocoques donnent des réactions différentes. Quand on traite le lait par le bleu de méthylène pour l'épreuve de la réductase, pour les laits certifiés et de haute qualité, on doit se souvenir que le bleu de méthylène n'est pas réduit ordinairement par le streptococcus mastitidis. Cependant la non-réduction du lait au bleu de méthylène n'est pas toujours constante: la concentration du colorant peut parfois n'être pas la même dans toutes les préparations commerciales.

\section{HÉmOLYSE}

I Un caractère d'espèce du streptocoque de la mammite que nous décrivons ici, est de ne pas être hémolytique. Il se produit parfois des changements de sang du type $\mathrm{G}$ ou $\mathrm{A}$ selon la classification de Brown, mais le streptocoque désigné sous le nom de Str. mastitidis ou agalactice, présentant les caractères décrits ici, n'est jamais $B$. hémolytique.

\section{FERMENTATION DES SUCRES}

Nous avons étudié le pouvoir de fermentation des hydrocarbones que nous considérons seulement de relative importance dans la différenciation des streptocoques. Nous avons, en particulier, fait des preuves de la fermentation avec de l'eau peptonée et le sucre étudié, d'après la formule de HIss : avec du sérum-peptone plus de la teinture de tournesol, et en outre avec du bouillon de cheval ayant un $p H$ de 7,3 , additionné de sucre correspondant après enlèvement du sucre normal du bouillon de cheval (en laissant le colibacille s'y cultiver 12 à 24 heures et en neutralisant ensuite avant addition du sucre correspondant, puis en stérilisant de nouveau).

Voici les résultats que nous avons trouvés au point de vue fermentation :

Lactose +
Glucose +
Galactose +
Mannite - +
Raffinose -
Levulose +
Inuline -
Maltose +

Saccharose $f$
Amidon avec un dépôt de
couleur orange.
Salicine + -
Xylose -
Arabinose -
Sorbite -
Trehalose +

Le clivage de I'hippurate de sodium. - Une des caractéristiques du streptococcus mastitidis est sa force de clivage de l'hippurate de sodium. Sous ce rapport, il diffère des streptocoques d'origine humaine, qui ont une action très faible ou nulle sur ce sel, qui selaisse difficilement dédoubler par eux. 
Le $\mathrm{pH}$ final. - Le $p \mathrm{H}$ final du streptococcus mastitidis dans le lactose et le sucrose après 48 heures d'incubation se trouve réduit à 4,4 ou à 4,6 . Les streptocoques d'origine humaine et animale ont un $p \mathrm{H}$ final plus élevé.

Pathogénéité. - J'ai injecté, en différentes occasions, 5 à $40 \mathrm{em}^{3}$ de culture du streptocoque de la mammite, avec les précautions aseptiques ordinaires, dans le canal du trayon et dans le sinus de vaches saines. La mammite s'est développée en 24 à 48 heures dans tous les quartiers injectés, et, dans un cas, elle fut purulente et d'un caractère aigu. J'ai aussi injecté 1,2 et $4 \mathrm{~cm}^{3}$ de lait provenant de pis malades infectés par du $S$. mastitidis et j'ai pu reproduire ainsi les symptômes de la mammite, qui ont persisté pendant les deux ou trois mois qui suivirent. Par contre, en frottant avec des cultures de streptocoques les orifices des trayons de 6 vaches saines, je n'ai pu produire la mammite. En vue de vaccination préventive, j'ai injecté intramusculairement des cultures virulentes de streptocoques à raison de 20 à $100 \mathrm{~cm}^{3}$ et, dans deux cas, de plus de $300 \mathrm{~cm}^{3}$, et je n'ai pu observer ni inflammation locale ni réaction générale.

Je n'ai pu, non plus, observer de virulence, à la suite d'injections intramusculaire et sous-cutanée, chez les rats, les cochons d'Inde, et les moutons, même avec des quantités plus grandes que les doses ordinaires expérimentales. Chez quelques cochons d'Inde femelles, chez lesquelles des injections sous-cutanées n'avaient pas produit de réactions marquées, les streptocoques de la mammite, injectés à travers la peau dans le parenchyme mammaire, ont toujours produit, après 2 ou 4 jours, une forte induration qui a persisté plus d'un mois. Il apparaît très elairement que le streptocoque de la mammite n'a de pathogénéité spécifique que dans les tissus glandulaires de la mamelle, et que, pour être virulent, il doit arriver à l'intérieur de la glande.

La pathogénéité du streptocoque de la mammite chez l'homme n'a jamais encore été démontrée. La plupart des auteurs qui ont travaillé sur le streptocoque de la mammite nele considèrent pas comme pathogène pour l'homme.

Les caractères morphologiques et culturaux des races du streptococcus mastitidis que j'ai étudiées au Canada, sont fondamentalement les mêmes que ceux des races que j'ai étudiées autrefois dans les différentes parties de l'Europe.

D'après les études comparatives des différents types de streptocoques isolés des animaux ou de l'homme, le streplocoque de la mammite doit être considéré comme une espèce ou variété spéciale.

No us devons cependant tenir compte, comme on l'a déjà mentionné, que des caractères plus ou moins divergents de ceux consi- 
dérés comme typiques, sont souvent le fait de certaines souches de streptocoques considérés comme formes atypiques, et que des cultures pratiquées longtemps sur les milieux artificiels usuels peuvent changer quelques-unes des réactions culturales ordinaires.

\section{QUELQUES OBSERVATIONS AU SUJET DES STREPTOCOQUES}

Les streptocoques comptent parmi les groupes de microorganismes les plus étudiés. Malgré tout cela et malgré les connaissances qu'on a pu réunir sur ces microorganismes, on est encore loin d'être satisfait. Nous ne pouvons pas encore accepter comme définitives, les données de la taxonomie sur la classification et la différenciation que les ouvrages de bactériologie nous fournissent sur les streptocoques.

Le système de toute la classification de nos livres de bactériologie est basé uniquement sur les connaissances nouvelles que l'on a eues de cette science, à mesure qu'elle progressait. Les premières classifications bactériologiques étaient basées sur la morphologie. Plus tard, les études biologiques des bactéries dans les différents milieux de culture, fournirent une base pour établir les qualités physiologiques semblables ou dissemblables entre les différents groupes de bactéries. Toujours est-il qu'on n'est pas encore complètement d'accord aujourd'hui sur la classification des groupes bactériologiques, parce qu'on ne s'entend pas sur la valeur des systèmes de classification. On ne s'accorde pas sur l'importance à donner aux réactions provoquées par les bactéries dans un groupe spécial de milieux de cultures, de préférence à d'autres réactions obtenues dans d'autres milieux. Quelques bactériologistes basent leur système de classification pour la détermination de certaines espèces, surtout sur l'action des microorganismes sur les sucres et les hydrocarbones en général, tandis que d'autres font autrement. Encore une fois, l'accord est loin d'être complet.

En ce qui concerne tout spécialement le streptocoque de la mammite, par exemple, on se trouve devant le fait, que les bactériologistes qui, dans les dernières années, ont étudié ce microorganisme dans ses réactions culturales, telles que nous les avons exposées dans ce travail, ont pu faire une différenciation plus précise du $S$. mastitidis des autres groupes de streptocoques que les chercheurs s'étant basés seulement sur l'hémolyse et les sucres. Grâce aux réactions en lait tournesolé, en lait au bleu de méthylène, en bouillon lactosé, en agarsang, sur l'hyppurate de soude, le $p \mathrm{H}$ final, la température du développement et la coagulation du lait, ete., on a pu faire une séparation plus précise qu'avec les réactions qui s'en tiennent simplement dे l'action de ces microorganismes sur les hydrates de carbone et à leur pouvoir hémolytique. Les auteurs mêmes des grands manuels bactériologiques très à jour (manuels classiques entre toutes les 
mains), ne traitent pas, exception faite de quelques ouvrages tout récents, dans le détail que mérite le sujet, le streptocoque de la mammite. L'on ne peut pas se servir de ces ouvrages pour avoir une caractérisation spécifique suffisante pour le différencier des autres germes semblables.

On ne devrait considérer comme la cause de la mammite que les streptocoques dont la culture, faite à même le lait ou son sédiment, provient d'un lait prélevé aseptiquement du pis de la vache. Le lait ordinaire peut renfermer des streptocoques de plusieurs sources, s'il n'est pas pris aseptiquement : des sécrétions du vagin, des fèces, du nez, de la bouche, etc. ; des sécrétions d'animaux voisins pouvant contenir des streptocoques de différentes sortes capables de contaminer le lait.

Le S. lacticus se trouve, sans exception, dans tous Jes laits, même s'ils sont pris avec toutes les précautions possibles de propreté, comme cela se fait pour le lait certifié, mais on ne le trouve pas dans un lait prélevé aseptiquement dans un récipient stérile, directement du trayon $d u$ pis de la vache. On peut en dire autant des streptocoques de différents animaux et de l'homme, hôtes habituels de la peau, de la gorge, des cavités nasales, qu'on trouve aussi dans le fumier, qui peut facilement ensuite contaminer le lait, excellent milieu de culture pour ce germe.

A notre point de vue, l'on ne peut dire qu'un type de streptocoque est l'agent causal de la mammite s'il ne provient d'un lait prélevé aseptiquement.

\section{FORMES ATYPIQUES DU STREPTOCOCCUS MASTITIDIS}

Ce serait une exception aux faits généraux de la bactériologie du streptocoque de la mammite que nous venons de décrire, s'il ne se présentait pas des variétés ou formes atypiques.

Elles ont été décrites presque par tous ceux qui se sont occupés de l'étude de ces microorganismes. Les plus importantes, selon nous, sont les suivantes : quelques souches du streptocoque coagulent le lait tournesolé sans présenter la réduction qu'ordinairement on voit au fond du tube, toute la colonne de lait demeurant rouge ; d'autres fois, par contre, la réduction se fait dans la plus grande partie de la colonne de lait, prenant un aspect de coagulation semblable à celui que produit le streptocoque lactique. Le lait au bleu de méthylène, qui ordinairement n'est ni réduit ni coagulé, réagit contrairement dans certains cas. Les cultures en bouillon lactosé restent souvent troubles, et le dépôt est moins floconneux, plus granuleux que dans les formes ordinaires, ressemblant alors aux cultures du streptococcus fecalis. Beaucoup de souches fermentent la salicine et très peu fermentent la mannite. 
On voit des différences dans la capacité de dédoublement de l'hyppurate de soude et dans la valeur du $p \mathrm{H}$ final. Bien que les cultures du streptocoque de la mammite n'aient aucune action hémolytique, quelques souches, en culture jeune, ont montré ce pouvoir hémolytique sur quelques types de sang, surtout sur sang de veau et sang humain, pendant qu'elles étaient sans action nette sur le sang de cheval.

Les cultures vieilles, quand elles sont passées sur de nouveaux milieux de cultures, donnent des réactions souvent assez différentes de celles des cultures jeunes. On note souvent que les cultures vieilles, repiquées en bouillons de différentes compositions, ont une tendance à laisser le bouillon trouble, contenant moins de sédiment au fond, et sont portées à former des chaînes plus courtes ou à prendre le type en diplocoques ou en amas. D'ailleurs l'aspect de la culture en bouillon dépend aussi de la composition du bouillon. De même, de rares souches, qui fermentaient la salicine et la mannite, peuvent avoir perdu ce pouvoir, après plusieurs passages en milieux de cultures. L'aspect et surtout les dimensions des colonies dans les différents agars, que nous avons nommés, changent parfois, en devenant plus petits, par repiquages successifs. Des souches fraîches montrent quelquefois des colonies ayant des dimensions plus grandes qu'à l'ordinaire. Une même culture donne parfois en cultures successives des colonies d'aspect et de dimensions variables.

Ces variations pourraient trouver une explication facile, si l'on admettait le fait, reconnu tous les jours davantage en bactériologie, de l'instabilité ou de la variabilité, dans certaines circonstances, des caractères phénotypiques ou acquis des bactéries. Cela est vrai surtout de certains types de bactéries. On reconnaît de plus en plus les faits de la mutation bactérienne par rapport à leurs caractères non génériques. Certaines études ont été effectuées et font reconnaitre déjà ces variations ou dissociations bactériennes dans de nouveaux groupes de microorganismes.

Nous sommes de jour en jour plus convainou que le streptocoque de la mammite chronique est un microorganisme ayant une parenté avec les bactéries du groupe lactique et le streptococcus fecalis, qu'on reconnaît par ailleurs comme ayant les plus grandes relations de parenté avec le streptococcus lactis. Il présente aussi de la ressemblance avec les microcoques blancs, appelés saprophytes de la mamelle. Les dissociations de caractères que nous avons obversées, en bien des reprises, dans les cultures que nous avons été obligé de considérer comme pures, ont fourni le plus souvent des aspects culturaux et morphologiques semblables à ceux des streptocoques lactis et fecalis et aux microcoques. Mais très souvent, ces effets apparents de mutation ou de dissociation bactérienne peuvent être dus à la présence de 
microcoques dans les colonies de streptocoques, chose assez fréquente, ou même à la présence d'autres types de streptocoques. Cette contamination, parfois difficile à découvrir et à éliminer, peut se continuer à travers différentes générations de germes en cultures successives et donner lieu à des interprétations fausses sur le caractère de telle ou telle souche. Il est certain qu'une pluralité de types de streptocoques, dans une colonie, n'est pas facile à découvrir par l'aspect de ces colonies.

Cependant, les études que nous avons faites à notre laboratoire, avec toutes les garanties désirables pour avoir la certitude de travailler avec des cultures pures de streptocoques, nous ont mis en évidence ce fait, que chez le streptocoque de la mammite repiqué en cultures successives, certains individus d'une souche peuvent prendre différents aspects culturaux et les perdre.

Ces manifestations de dissociation et des formes filtrables trouvées dans des souches de nos streptocoques, que nous étudierons dans un travail prochain, peuvent nous aider à comprendre plusieurs choses encore imprécises dans l'apparition de la maladie.

Nous voulons, ici, seulement dire que nous croyons devoir être persuadé que le streptocoque de la mammite (streptococcus mastitidis str. agalactice) présente des formes de dissociation donnant origine à des formes filtrables et à des formes qui ne peuvent pas se différencier des microcoques. De même, pour l'aspect morphologique, des individus différents d'une même chaîne streptococcique se présentent, relativement souvent, sous la forme de bâtonnets d'aspect diphtéroïde, comme, également, des individus d'une même chaîne montrent une division en différents plans telle qu'elle constitue une caractéristique générique des microcoques.

\title{
RÉACTION DE SCHARDINGER. \\ LAITS CRUS COLOSTRAUX ET CONTROLE HYGIÉNIQUE A LA CONSOMIMATION
}

\author{
par
}

\section{A. HOUDINIÈRE}

Docteur-Vétérinaire, Inspecteur des denrées alimentaires de la ville de Naney.

Le décret du 25 mars 1924 portant règlement d'administration publique pour l'application de la loi du Ier août 1905 sur la répression des fraudes en ce qui concerne le lait, précise, en son article 2, que :

"Ne peut-être considéré comme lait propre à la consommation humaine $: 1^{\circ} \ldots \ldots ; 2^{\circ} \ldots . ; 3^{\circ}$ le lait provenant d'une traite opérée 